\section{Progressive Transmission of Line Drawings Using the Wavelet Transform}

George Müller, Christiane Klöditz, Jan Bons, and Ramjee Prasad

Abstract-This paper presents a method to apply progressive transmission to line drawings using the wavelet transform. Experiments have been conducted and showed that the wavelet transform, combined with a quantization step, performs progressive transmission using a data rate comparable to standard chain coding at the expense of almost no visually perceptible distortion.

\section{INTRODUCTION}

The amount of data required to encode graphical information is very high compared to numerical and alphanumerical information. This makes storage of graphical information very expensive. Another consequence of this huge amount of data is the large bandwidth that is needed for the transmission of graphical information. A practical example can easily illustrate this difficulty. Suppose we have a digital picture of size $256 \times 256$ pixel, each pixel encoded with eight bits. If we want to transmit this image, line by line, over a narrowband channel such as a telephone line using a $1200 \mathrm{~b} / \mathrm{s}$ modem, it takes $7.28 \mathrm{~min}$ for the complete transmission of the digital picture. In order to reduce storage costs and transmission time, many techniques can be found in the literature [1], [2] that reduce the amount of data necessary to encode graphical information. Although the amount of data can be reduced efficiently using these techniques, it is essential to wait for the transmission to end before it is possible to recognize the contents of the graphic. This problem is not due to the amount of data, but by the rigid order in which the data is transmitted (line by line).

To alleviate this matter, progressive transmission [3] first sends a low-resolution approximation for recognition purposes. Then this low resolution approximation is refined progressively by further transmission. Or, in other words, progressive transmission hierarchically reorganizes the information of an image in such a way that early recognition becomes possible. Progressive transmission uses the image understanding of the human user. When a human is the end user of the transmitted image, this method of information reorganization of an image can result in extreme bandwidth savings. An user may know the general characteristics of the image he/she is looking for, and could thus make a positive decision about the image (want/don't want or stop/complete transmission) with much less information than required for a normal, sequentially scanned transmission at full resolution.

Applications of progressive transmission are, for instance, "telebrowsing" remote image databases, interactive graphics with a remote computer, and teleconferencing. Therefore, the authors got motivated to investigate progressive transmission of line drawings.

Until now, all research in progressive transmission has been concentrated in the field of 2-D images (e.g., photographs). Indeed, the usually more modest data rates for line drawings could be the reason why no techniques for progressive transmission of graphical representations of line drawings can be found in the literature. To the

Manuscript received November 28, 1993; revised July 7, 1995. The associate editor coordinating the review of this work and approving it for publication was Dr. Michael Unser.

The authors are with the Telecommunications and Traffic Control Systems Group, Delft University of Technology, Delft, The Netherlands.

Publisher Item Identifier S 1057-7149(96)02756-X.

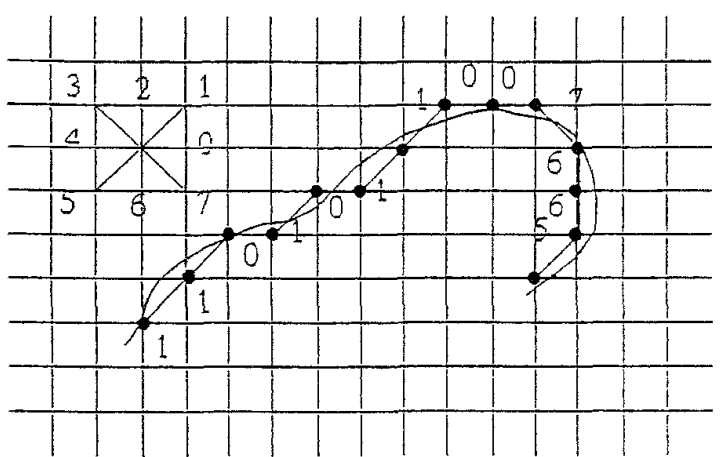

Fig. 1. Illustration of chain coding a curve.

authors' best knowledge, we are the first to propose to use progressive transmission for line drawings.

The application of progressive transmission for many existing line drawings, viz. engineering drawings, geographical maps, and contours extracted from grey-level images can be interesting, although they are encoded using considerable numbers of bits.

This paper gives the first insight into the application of progressive transmission for line drawings. Future work on this topic is recommended.

\section{LINE DRAWINGS}

Examples of line drawings are signatures, engineering drawings, and geographical maps. Clearly, they differ from other graphics, viz. photographs because they only consist of lines, curves, and text projected at a usually white background. Therefore, representing line drawings and progressive transmission of line drawings will also be different. One way to encode line drawings would be the use of run-length coding, which encodes sequences of black or white pixel and has found a practical application in digital facsimile systems [4]. Because this technique, however, also encodes the background, which does not carry any information, run-length coding is not a very efficient way to encode line drawings. It is found that chain coding is a more efficient way for line drawings [5]. Chain coding, pioneered by Freeman [6] in the 1960's, approximates the curves of a line drawing by a set of straight line segments. The quantization step is carried out by overlaying the original line drawing with a square lattice and connecting the lattice nodes closest to the original line drawing with straight line segments. If we regard these line segments as being vectors pointing from one node to the following node, eight different directions are possible. Freeman proposed to encode these vector strings by encoding the absolute coordinates of the starting node of each curve and encoding the directions of all following vectors in the string using a coding ring. An encoding example is illustrated in Fig. 1. A drawback of chain coding is its sensitivity to transmission errors; when one segment is incorrectly received, the remainder of the drawing will be placed at a wrong position. Another possible method that is less susceptible to errors is to represent line drawings by means of lists of sample point coordinate pairs.

After reviewing the various techniques to perform progressive transmission of grey-level images [7], a good progressive coding technique should provide the following six features: i) use of previously sent data for the next approximation; ii) low correlation between successive transmissions; iii) low correlation between neighboring 


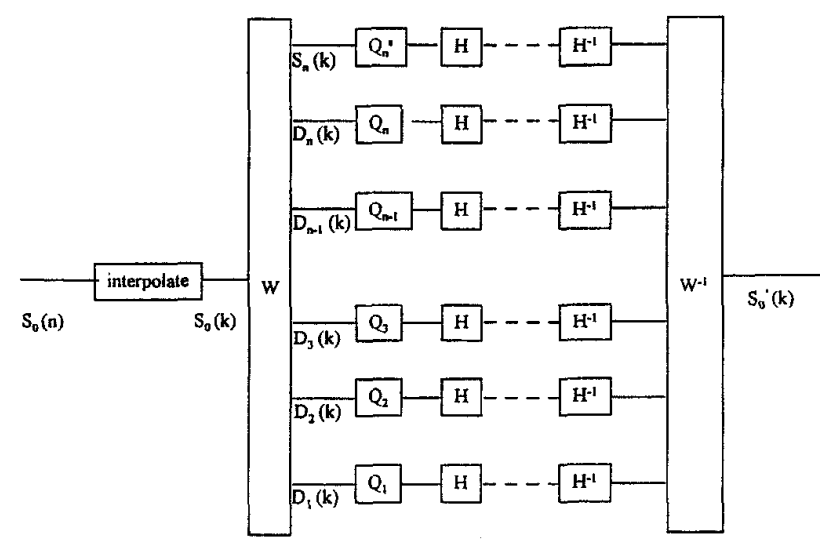

Fig. 2. Progressive transmission of line drawings using the wavelet transform.

pixels; iv) fast recognizability; v) lossless transmission; and vi) amount of data equal to or less than ordinary encoding.

\section{Progressive Transmission Using the Wavelet Transform}

In [8], a study is presented on the development of a hierarchical planar curve descriptor using the biorthogonal wavelet transform. This descriptor decomposes curves into components of different scales. The applications discussed include character recognition and shape deformation, and show the invariance, uniqueness, and stability properties of the descriptor. A comparison of curves reconstructed by Fourier descriptors and wavelet descriptors proved that the Fourier descriptor is not efficient in describing shapes with only local deformation. A small perturbation of one parameter of the Fourier descriptor will affect the entire boundary of a shape. However, the wavelet descriptor provides a scheme to model local and global deformation. That is why we decided to study the performance of the wavelet transform for progressive transmission, and Fig. 2 illustrates the way it was implemented. The original signal $\boldsymbol{S}_{0}(\boldsymbol{n})$ on the lefthand side represents the coordinate pairs of a line drawing. After a linear interpolation step, the now equidistant samples are wavelet transformed in block $W$. This results in the lowest resolution signal $S_{n}(k)$ and the difference signals $D_{1}(k) \cdots D_{n}(k)$. After this the lowest resolution signal $\boldsymbol{S}_{n}(\boldsymbol{k})$ and as many difference signals as wanted by the receiver are quantized by the quantizers $\boldsymbol{Q}_{s}^{n} \cdots \boldsymbol{Q}_{1}$, Huffman encoded by the coders $\boldsymbol{H}$ and transmitted to the receiver. At the receiver these signals are decoded by the decoders $\boldsymbol{H}^{-1}$ and reconstructed by $\boldsymbol{W}^{-1}$ to the approximation $\boldsymbol{S}_{0}^{\prime}(\boldsymbol{k})$. Because the line drawings used were created by a writing tablet or by contour-extracted bitmap graphics, the sample coordinates were not equally spaced. However, the wavelet transform expects equidistant samples. Therefore, a linear interpolation step was performed producing equidistant samples for both $x$ and $y$ coordinates.

The wavelet transform can be looked at as a multiresolution decomposition of $x$ and $y$ samples [9]. This decomposition consists of the initial approximation and the detail signals. The wavelet transform was carried out by recursively lowpass and highpass filtering, resulting in decreasing resolution versions of the $x$ and $y$ coordinate signals. The used quadrature mirror filters $H(\omega)$ and $G(\omega)$ consist of four coefficients, first recognized and solved by Daubechies [10]. The coefficients of $H(\omega), h_{0}, h_{1}, h_{2}$ and $h_{3}$ are given by

$$
\begin{array}{ll}
h_{0}=\frac{1+\sqrt{3}}{2} & h_{1}=\frac{3+\sqrt{3}}{2} \\
h_{2}=\frac{3-\sqrt{3}}{2} & h_{3}=\frac{1-\sqrt{3}}{2} .
\end{array}
$$

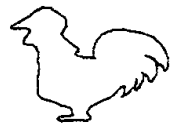

(a)

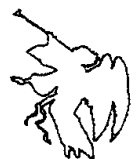

(b)

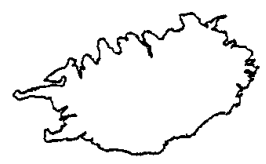

(c)

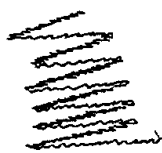

(d)

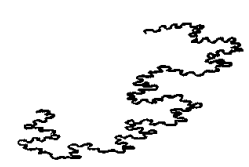

(e)
Fig. 3. Used sample line drawings: a) Haan (1024 samples); b) Engel (1024 samples); c) IJsland (1024 samples); d) Zig Zag (4096 samples); e) Fractal (4096 samples).

For the boundary points, a circular symmetry is assumed, which gives the lowest distortion for closed shapes. Line crossings and endings have not been treated in a special way, so a small deterioration will occur at the endings dependent on the size of the filter.

The wavelet transform has turned the original coordinate signals $x(k)$ and $y(k)$ of the line drawing into two columns of floating point numbers. Clearly, the direct transmission of these columns will take a long time to complete. To obtain a data rate comparable to that of standard chain coding, data compression techniques can be used. Quantization, combined with an efficient bit allocation and an appropriate encoding strategy (Huffman encoding) can accomplish this reduction task at the expense of a distortion unnoticeable to the human eye [11]. As far as quantization is concerned, the process can be split into three steps: i) statistical measurements; ii) histogram fitting; and iii) Max-Lloyd quantization.

Statistical Measurements: In order to construct an efficient quantization scheme, we have measured the statistics of five test line drawings, see Fig. 3. Three of them, Fig. 3(a)-(c), were created using the writing tablet; the other two (Fig. 3(d) and (e)) were the result of contour tracing bitmap images. Of all wavelet subbands, (the different $D$ - and $S$-signals), the histogram normalized with respect to the variance of the subband was computed. All the resulting histograms appeared to have the same shape, except for the subband containing the $S$ coefficients. For the $D$ signals, it was therefore possible to compute one normalized histogram that represents the statistics of all $D$ frequency channels.

Histogram Fitting: In order to have a mathematical description of the measured histogram, it is essential to find a known mathematical description that resembles our histogram sufficiently close. One such description is the generalized Gaussian probability density function (PDF), as follows:

$$
\begin{aligned}
& p(x)=a e^{-|\boldsymbol{b x}|^{c}} \quad \text { with } \\
& a=\frac{b c}{2 \boldsymbol{\Gamma}\left(\frac{1}{c}\right)}, \quad b=\frac{1}{\sigma_{x}} \sqrt{\frac{\Gamma\left(\frac{3}{c}\right)}{\Gamma\left(\frac{1}{c}\right)} .}
\end{aligned}
$$

Note two special cases for the parameter $c$. For $c=1.0$, we have the Laplacian PDF, and for $c=2.0$, we have the well-known Gaussian PDF. The chi-squared test was applied for the fitting of the data of the measured normalized histogram to the generalized 


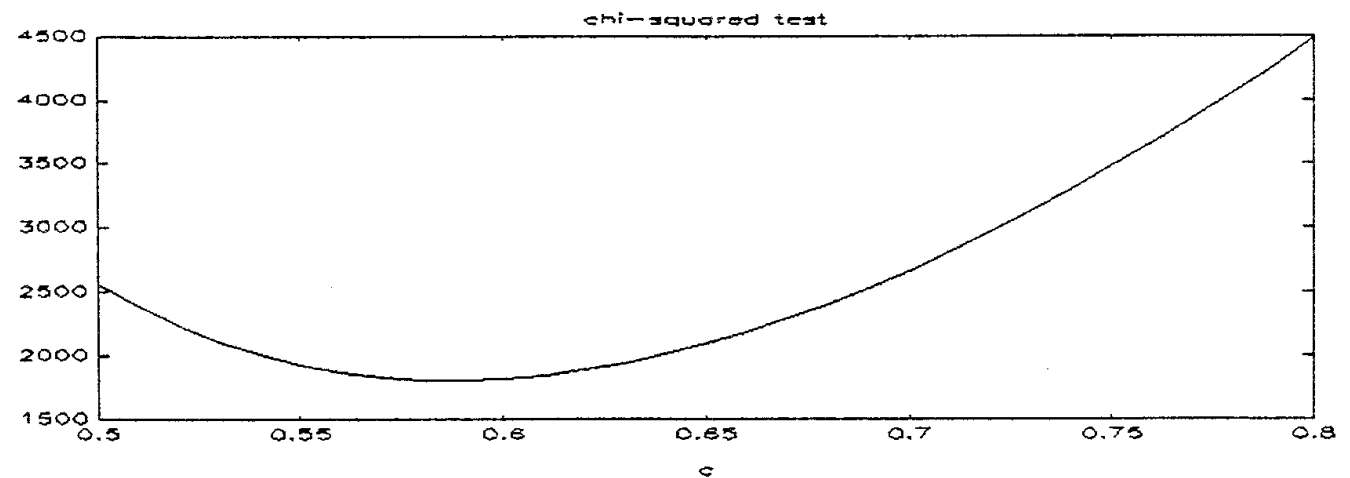

(a)

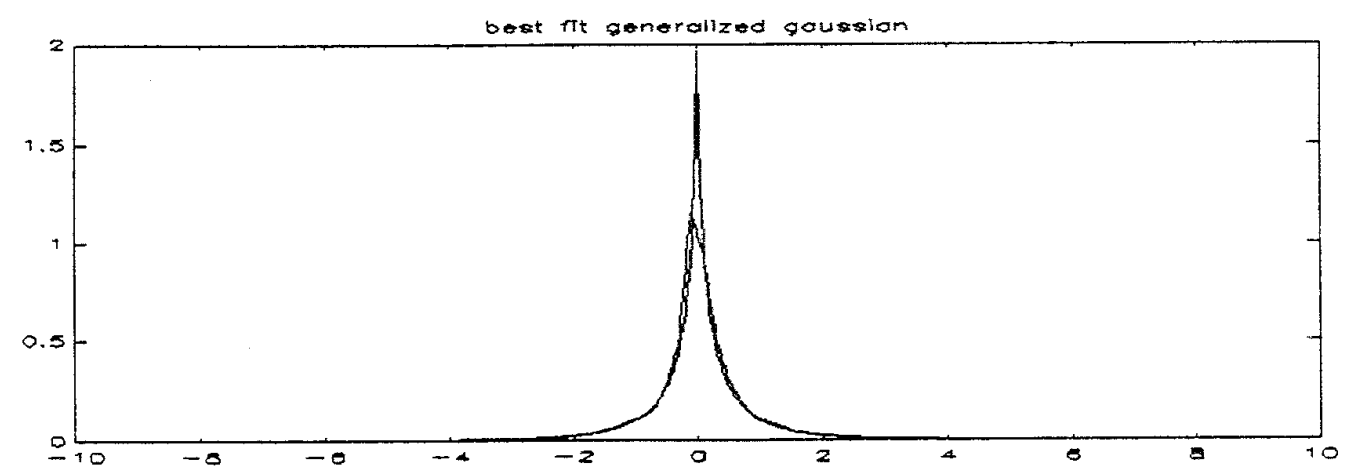

(b)

Fig. 4. Fitting the generalized Gaussian PDF to the normalized histogram of the $c$ signals: a) chi-squared test; b) best fit: $c=0.5$.

Gaussian density function. This test returns the chi-squared test statistic $t_{\chi^{2}}$ as a measure of the fit of the sample density function $f_{X}(\cdot)$ consisting of the samples $X=\left[x_{1}, x_{2}, \cdots, x_{M}\right]$, and the given density function $f(\cdot)$, as follows:

$$
t_{\chi^{2}}=M \sum_{i=1}^{M} \frac{\left[f_{X}\left(x_{i}\right)-f\left(x_{i}\right)\right]^{2}}{f\left(x_{i}\right)} .
$$

The results of the chi-squared statistic for generalized Gaussian PDF's computed with parameter $c$ ranging from 0.5 to 0.8 are illustrated in Fig. 4(a). Obviously, the minimum, which corresponds with the best fit, lies somewhere between 0.5 and 0.6. In Fig. 4(b) we see how closely the generalized Gaussian with $c=0.5$ fits the normalized histogram.

As mentioned earlier, only the $D$ wavelet signals obtained by filtering with filter $g(n)$ have histograms corresponding to the generalized Gaussian PDF with $c=0.5$. The $S$ signals, representing the coarsest approximation of the line drawing with only 16 coordinate pairs, have a more-or-less random density. Taking differences between successive coefficients and then computing the histogram of these difference coefficients, a histogram that is relatively the same for all $S$ signals of the various line drawings is acquired. Applying the chi-squared test on these histograms, an optimum value for $c=2.0$ (see Fig. 5(a)) can be found, which is the well-known Gaussian PDF. Therefore, it is possible to conclude that the fit of the difference coefficients histogram to the Gaussian PDF is reasonable (see Fig. 5(b)). Resuming, the generalized Gaussian with $c=0.5$ for all $D$ signals can be used; whereas the differential $S$ signals can be modeled with the specific Gaussian PDF.
Max-Lloyd Quantization: Note that there are models to represent the statistics of the various wavelet coefficients. The Max-Lloyd approach [12] can be used to construct optimum quantizers. Therefore, optimum decision levels are given by

$$
\begin{aligned}
X_{1, \mathrm{opt}} & =-\infty \\
x_{k, \mathrm{opt}} & =\frac{1}{2}\left(y_{k, \mathrm{opt}}+y_{k-1, \mathrm{opt}}\right), \quad k=2,3, \cdots, L \\
x_{L+1, \mathrm{op} t} & =\infty .
\end{aligned}
$$

The corresponding representation levels are given by

$$
y_{k, \mathrm{opt}}=\frac{\int_{x_{k, \mathrm{opt}}}^{x_{k+1, \mathrm{opt} t}} x p_{x}(x) d x}{\int_{x_{k, \mathrm{opt}}}^{x_{k+1, \mathrm{opt}}} p_{x}(x) d x} \quad k=1,2, \cdots, L .
$$

The more levels a quantizer has, the more bits are needed to encode the quantizer's output. How many bits have to be assigned to each quantizer in order to minimize the total distortion for a specified data rate? To answer this question, it is assumed that the total distortion rate $D(v)$ and the total data rate $R(v)$ can be considered as being sums of the individual distortion rates $d(v)$ and individual data rates $r(v)$ of each quantizer $v$. The problem is finding the optimal vector $v$ that minimizes the overall distortion for a certain data rate. Westerink [11] described an algorithm using a parameter

$$
s\left(v_{n}, k_{n}\right)=\frac{r_{n}\left(v_{n}\right)+r_{n}\left(k_{n}\right)}{d_{n}\left(v_{n}\right)-d_{n}\left(k_{n}\right)}
$$




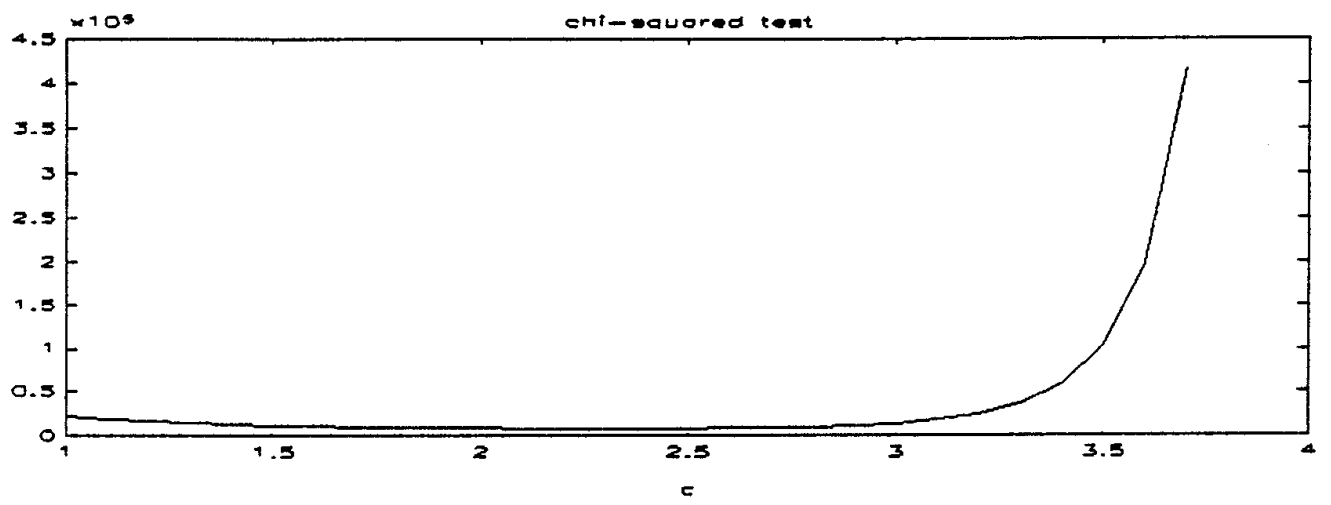

(a)

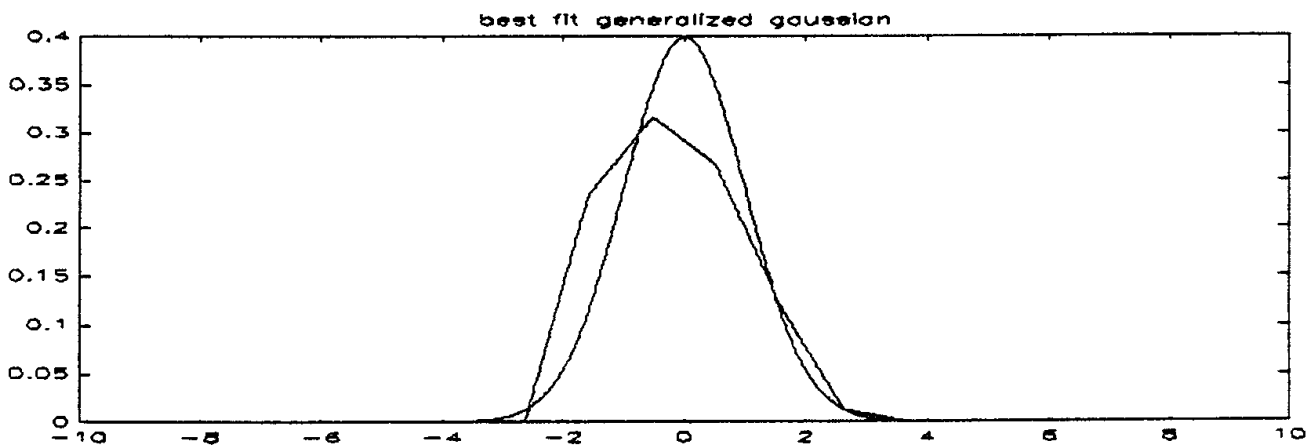

(b)

Fig. 5. Fitting the generalized Gaussian PDF to the normalized histogram of DPCM-encoded $s$ coefficients: (a) chi-squared test; (b) best fit: $c=2.0$.

TABLE I

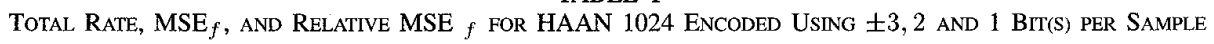

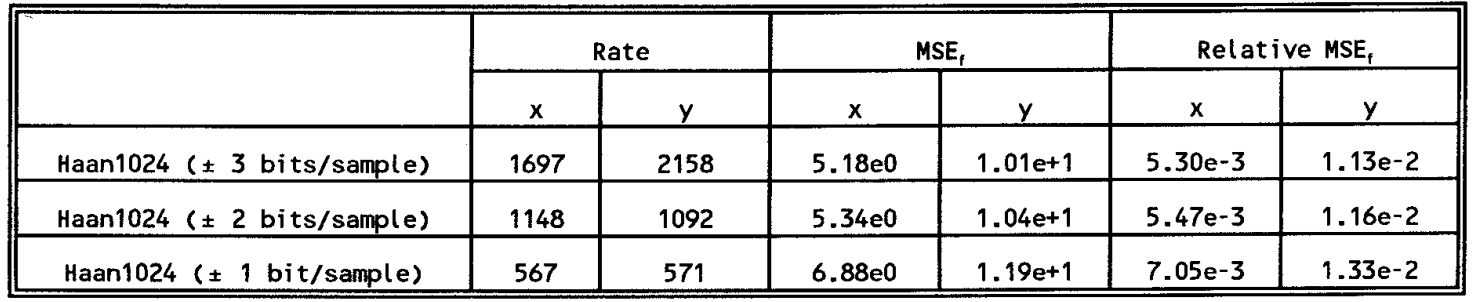

as a measure of efficiency. The larger the measure, the more efficient is the allocation of quantizer $v_{n}$ to frequency band $n$. Because the described algorithm to construct the quantizers does not assume integer number of bits, the representation levels of the quantizers can be encoded with variable-length codes (Huffman code). The principle of the Huffman code is to assign long codewords to quantization levels with a low probability of occurrence and to assign short code words to those levels having high probability of occurrence.

\section{RESULTS AND Discussions}

Fig. 6 shows the original sample line drawings overlaid with the final reproductions coded using three, two and one b/sample. Three b/sample is approximately the same rate as that of standard chain coding. As to be seen, shapes containing many high-frequency components (Zig Zag and Fractal images) have larger distortions when encoded using two b/sample and one b/sample. This can be explained by the fact that the quantization step regards high-frequency wavelet coefficients as less important than low-frequency ones. A more quantitative illustration of the performance of the system is given in Tables I-V. In these tables, the total rate necessary to encode the final approximations, the mean square error (MSE) of a signal $f(n)$ defined by

$$
\operatorname{MSE}_{f}=E\left[\left(f_{\text {original }}(n)-f_{\text {reconstructed }}(n)\right)^{2}\right]
$$

and the relative $\mathrm{MSE}_{f}$ expressed by

$$
\text { relative } \operatorname{MSE}_{f}=\frac{E\left(f_{\text {original }}(n)-f_{\text {reconstructed }}(\dot{n})\right)^{2}}{E\left(f_{\text {original }}(n)\right)}
$$

are given for an average number of $b /$ sample of three, two and one.

In Fig. 7, a comparison between normal transmission using standard chain coding and progressive transmission using the wavelet 

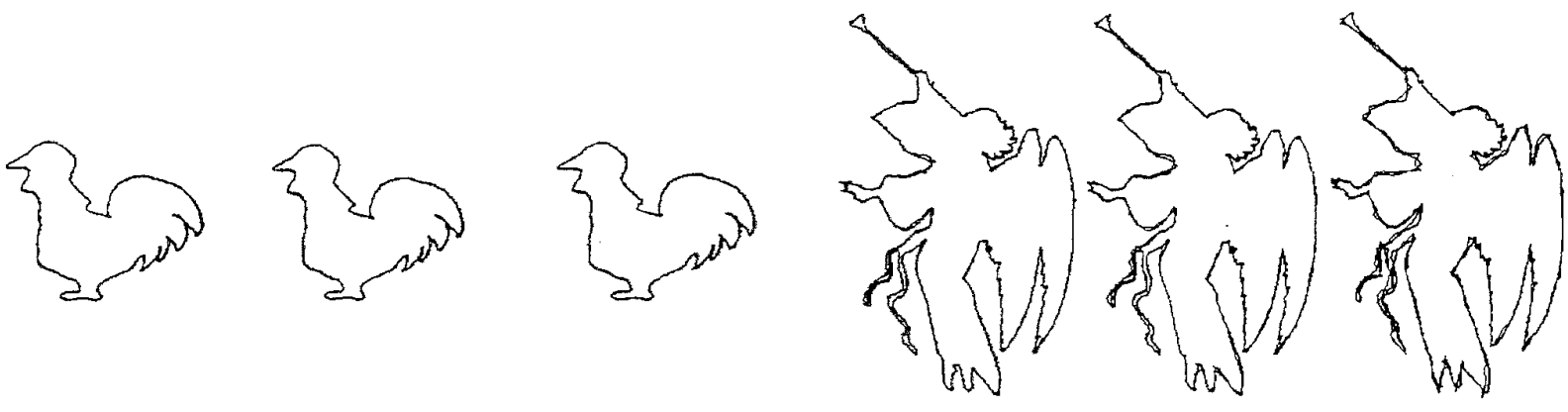

(a)
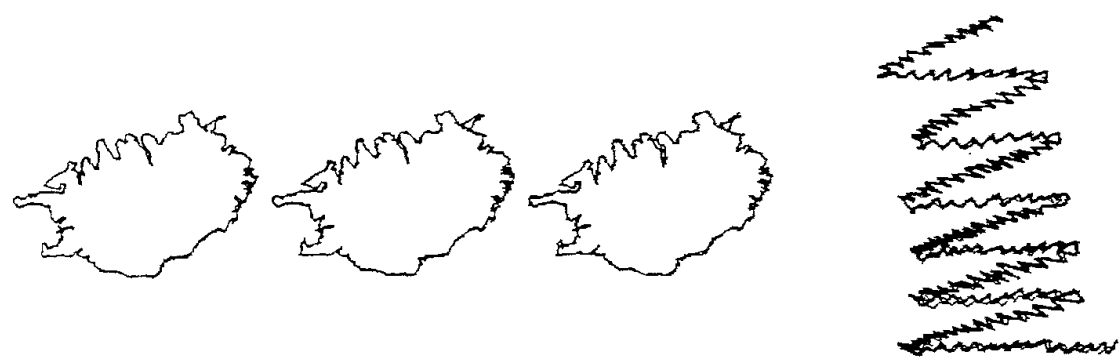

(b)
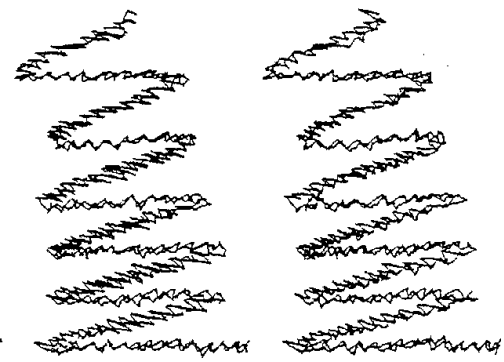

(c)

(d)

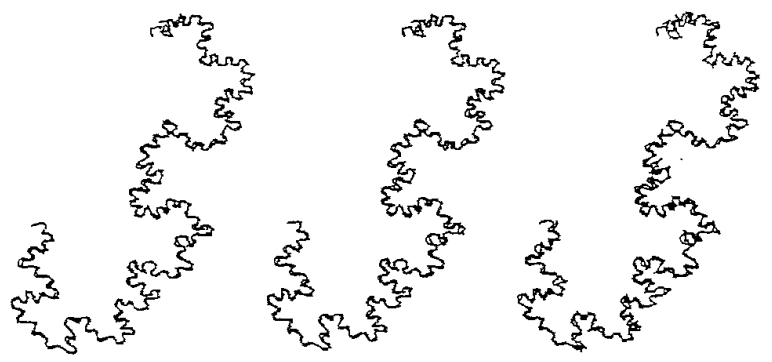

(e)

Fig. 6. Reconstruction results for 3, 2, and 1 b/sample. (a) Haan. (b) Engel. (c) IJsland. (d) Zigzag. (e) Fractal.

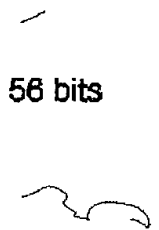

441 bits

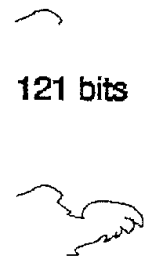

751 bits

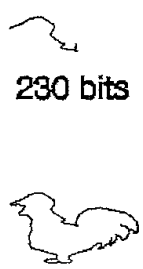

1091 bits

(a)

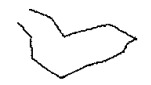

56 bits<smiles>CCCCCCCCCCCCCCCCCCC</smiles>

441 bits

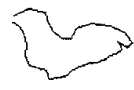

121 bits

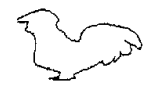

230 bits

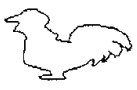

751 bits

(b)

Fig. 7. Comparison of (a) chain coding and (b) the wavelet transform, in terms of early recognizability.

transform method is given. For equal bit rates we can conclude that using the progressive transform method, the contents of the drawing can be recognized in an earlier stadium as is the case using normal transmission.

\section{CONCLUSIONS AND RECOMMENDATIONS}

We have presented an approach for progressive transmission of line drawings using the wavelet transform. Since the line drawings were represented by the coordinates of sample points, a quantization step is necessary to reduce the amount of data to the amount used by standard chain coding. When coding the original coordinates using $22 \mathrm{~b} /$ sample (resolution $2048 \times 2048$ ), this step yields a compression ratio of seven or more at the expense of an unnoticeable distortion. Recognition of the contents of the line drawing is possible right from the beginning of the transmission.

The following recommendations are made for future studies.

1) Application on different line drawings - The line drawings used for the experiments were relatively simple, each consisting of only one open or closed curve. Therefore, further research is recommended on multiple-curve line drawings with an arbitrary number of samplepoints as well as noncontrived line drawings. 
TABLE II

Total RATE, MSE $_{f}$, AND Relative MSE $f$ FOR ENGEL 1024 ENCOdEd Using $\pm 3,2$, ANd 1 Bit(S) PER SAMPLE

\begin{tabular}{|c|c|c|c|c|c|c|}
\hline & \multicolumn{2}{|c|}{ Rate } & \multicolumn{2}{|c|}{ MSE $_{1}$} & \multicolumn{2}{|c|}{ Relative MSE $_{f}$} \\
\hline & $x$ & $y$ & $x$ & $y$ & $x$ & $y$ \\
\hline Engel 1024 ( \pm 3 bits/sample) & 1565 & 2189 & $3.68 \mathrm{e}+2$ & $6.14 \mathrm{e}+2$ & $6.03 e-2$ & $1.66 \mathrm{e}-1$ \\
\hline Engel 1024 ( \pm 2 bits/sample) & 1181 & 1356 & $4.33 \mathrm{e}+2$ & $7.06 \mathrm{e}+2$ & $7.10 \mathrm{e}-2$ & $1.91 e-1$ \\
\hline Engel 1024 ( $\pm 1 \mathrm{bit} /$ sample) & 432 & 717 & $1.31 \mathrm{e}+3$ & $1.33 e+3$ & $2.15 e-1$ & $3.59-1$ \\
\hline
\end{tabular}

TABLE III

Total Rate, MSE $f$, ANd Relative MSE $f$ FOR IJSLAND 1024 Encoded Using $\pm 3,2$, ANd 1 Bit(S) Per Sample

\begin{tabular}{|c|c|c|c|c|c|c|}
\hline & \multicolumn{2}{|c|}{ Rate } & \multicolumn{2}{|c|}{$\mathrm{MSE}_{\boldsymbol{i}}$} & \multicolumn{2}{|c|}{ Relative MSE $_{f}$} \\
\hline & $x$ & $y$ & $x$ & $y$ & $x$ & $y$ \\
\hline $\begin{array}{c}\text { 1Jsland1024 ( } \pm 3 \\
\text { bits/sample) }\end{array}$ & 1702 & 2390 & $7.55 e+2$ & $1.97 \mathrm{e}+3$ & $1.20 \mathrm{e}-1$ & $4.42 e^{-1}$ \\
\hline $\begin{array}{c}\text { IJsi and1024 ( } \pm 2 \\
\text { bits/sample) }\end{array}$ & 609 & 1393 & $2.78 \mathrm{e}+3$ & $2.99 e+3$ & $4.30 \mathrm{e}-1$ & $6.71 e-1$ \\
\hline $\begin{array}{c}\text { IJsland } 1024 \text { ( } \pm \\
\text { bit/sample })\end{array}$ & 578 & 662 & $3.17 e+3$ & $3.93 e+3$ & $4.90 \mathrm{e}-1$ & $8.82 \mathrm{e}-1$ \\
\hline
\end{tabular}

TABLE IV

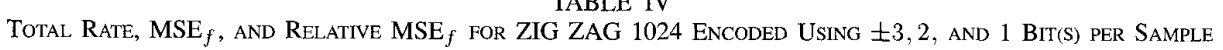

\begin{tabular}{|c|c|c|c|c|c|c|}
\hline & \multicolumn{2}{|c|}{ Rate } & \multicolumn{2}{|c|}{ MSE $_{f}$} & \multicolumn{2}{|c|}{ Relative MSE $_{\text {f }}$} \\
\hline & $x$ & $y$ & $x$ & $y$ & $x$ & $y$ \\
\hline Zigzag1024 ( \pm 3 bits/sample) & 2187 & 6410 & $3.23 e+1$ & $2.47 e+1$ & $2.85 e-2$ & $3.41 \mathrm{e}-3$ \\
\hline Zigzag1024 ( \pm 2 bits/sample) & 859 & 5074 & $2.26 e+2$ & $3.88 \mathrm{e}+1$ & $2.00 \mathrm{e}-1$ & $5.36 e-3$ \\
\hline $\begin{array}{c}\text { zigzag1024 ( } 1 \\
\text { bit/sample) }\end{array}$ & 302 & 2902 & $2.57 \mathrm{e}+2$ & $5.57 e+1$ & $2.27 \mathrm{e}-1$ & $7.70 e-3$ \\
\hline
\end{tabular}

TABLE V

Total RATE, MSE $_{f}$, ANd Relative MSE $f$ for FRACTAL 1024 Encoded Using $\pm 3,2$, and 1 Bit(S) Per SAMPLE

\begin{tabular}{|c|c|c|c|c|c|c|}
\hline & \multicolumn{2}{|c|}{ Rate } & \multicolumn{2}{|c|}{$\mathrm{MSE}_{\mathrm{f}}$} & \multicolumn{2}{|c|}{ Relative MSE $_{f}$} \\
\hline & $x$ & $y$ & $\mathbf{x}$ & $y$ & $x$ & $y$ \\
\hline $\begin{array}{c}\text { Fractal1024 ( } \pm 3 \\
\text { bits/sample) }\end{array}$ & 3037 & 5541 & $1.30 \mathrm{e}+1$ & $7.10 \mathrm{e} 0$ & $2.24 e-2$ & $1.03 e-2$ \\
\hline $\begin{array}{c}\text { Fractal } 1024 \text { ( } \pm 2 \\
\text { bits/sample })\end{array}$ & 1699 & 5022 & $2.13 \mathrm{e}+1$ & $1.24 \mathrm{e}+1$ & $3.67 e-2$ & $1.80 e^{-2}$ \\
\hline $\begin{array}{c}\text { Fractal } 1024 \text { ( } \pm \\
\text { bit/sample) }\end{array}$ & 999 & 2710 & $3.13 e+1$ & $2.41 e+1$ & $5.39 e-2$ & $3.51 \mathrm{e}-3$ \\
\hline
\end{tabular}

2) Comparison of rate-distortion performance-Tables of MSE results could be replaced by a graphical comparison of ratedistortion performance for chain coding and wavelet compression at a variety of bit rates and with different coding schemes (e.g., different filter banks, different subband decompositions, and different quantization strategies).

3) Experiments with other wavelet bases--The used wavelet bases are the smallest possible. Future research will include ex- periments with other wavelet bases and a comparison of the results.

4) Comparison with chain coding. A strong comparison with chain coding will be included in future work.

\section{ACKNOWLEDGMENT}

The authors are grateful to Prof. Dr. J. C. Arnbak for his interest and valuable suggestions. They also like to thank anonymous reviewers for their comments. 


\section{REFERENCES}

[1] A. K. Jain, "Image data compression: a review," Proc. IEEE, Mar. 1981, vol. 69 , no. 3, pp. 349-389.

[2] _ "Fundamentals of digital image processing." London, U.K. Prentice-Hall, 1989.

[3] S. L. Tanimoto, "Image transmission with gross information first," Comput. Graphics Image Processing, vol. 9, pp. 72-76, Jan. 1979.

[4] CCITT, "Standardization of group 3 facsimile equipment for document transmission," CCITT Recommendation T. 4.

[5] K. Liu and R. Prasad, "Comparing coding efficiency of vector chain coding and run-length coding for line drawings," Proc. Inst. Elect. Eng. $-I$, vol. 138 , no. 5 , pp. $363-370$, Oct. 1991.

[6] H. Freeman, "On the encoding of arbitrary geometric configurations," IRE Trans. Electron. Comput., vol. EC-10, pp. 260-268, June 1961.

[7] K. H. Tzou, "Progressive image transmission: a review and comparison of techniques," Opt. Eng., vol. 26, no. 7, pp. 581-589, July 1987.

[8] C.-H. Chuang and C.-C. Jay Kuo, "Multiscale planar curve descriptor using biorthogonal wavelet transform," Visual Inform. Processing II, SPIE vol. 1961, pp. 44-54, 1993.

[9] S. G. Mallat, "A theory for multiresolution signal decomposition: the wavelet representation," IEEE Trans. Pattern Anal. Machine Intell., vol. 11, no. 7, pp. 675-69, July 1989.

[10] I. Daubechies, "Orthogonal bases of wavelets with finite supportconnections with discrete filters," in Proc. Int. Conf.: Wavelets: TimeFrequency Methods and Phase Space, (J. M. Combes, A. Grossmann, Ph. Tchamitchian, Eds.,) Berlin, Germany: Springer, 1987.

[11] P. H. Westerink, "Subband coding of images," Ph.D dissertation, Delft Univ. Technology, Delft, The Netherlands, 1989.

[12] N. S. Jayant and P. Noll, Digital Coding of Waveforms. Englewood Cliffs, NI: Prentice Hall, 1984.

\section{Epipolar Line Estimation and Rectification for Stereo Image Pairs}

\author{
D. V. Papadimitriou and T. J. Dennis
}

Abstract-The assumption that epipolar lines are parallel to image scan lines is made in many algorithms for stereo analysis. If valid, it enables the search for correspondent image features to be confined to one dimension and, hence, simplified. An algorithm that generates a vertically aligned stereo pair by warped resampling is described.

\section{INTRODUCTION}

The centers of projection of the two cameras of a stereo viewing arrangement and any point in their field of view define an epipolar plane. The intersection of any such plane with the left or right image plane generates a line in 2-D image space called an epipolar line [1]. Thus, corresponding points of the two images belong to corresponding epipolar lines. This geometric relationship is called the epipolar constraint. Many stereo matching algorithms make the assumption that conjugate epipolar lines are collinear (or nearcollinear), which enables them to restrict the search for homologous image points to one dimension [2]-[4]. The algorithm hence executes faster and, when the assumption is valid, gives more accurate results. When the application for the use of stereo is to compute absolute

Manuscript received April 24, 1994; revised May 28, 1995. The associate editor coordinating the review of this paper and approving it for publication was Prof. John Juyang Weng.

The authors are with the Department of Electronic Systems Engineering, University of Essex, Colchester, U.K. (e-mail: tim@essex.ac.uk).

Publisher Item Identifier S 1057-7149(96)02764-9.

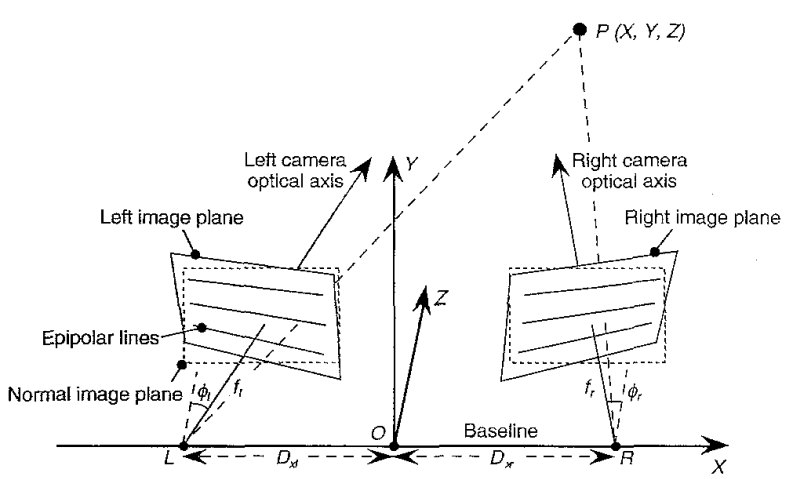

Fig. 1. Convergent stereo camera geometry. $P$ represents a general world-point. With this camera arrangement the sets of epipolar lines are not parallel. Dashed lines represent the image planes after rectification.

3-D information about a scene, then camera calibration data must be available, and the use of the epipolar constraint relies on a simple geometrical computation. However, there are other applications [5], such as object recognition and photogrammetry, where accurate calibration is unnecessary or difficult to obtain and where the epipolar constraint has to rely on assumptions about the camera geometry. The present work aims to provide a solution for the latter case.

As may be inferred from the above, stereo disparity analysis may be facilitated by first producing a vertically aligned stereo pair and then applying to it an algorithm that performs a 1-D search along the image scan lines in order to establish a dense field of point correspondences.

There is extensive literature on the reconstruction of a scene and/or the determination of object or camera motion from point correspondences between two views [6]-[9]. However, the task of establishing the transformations for rectification of the images is not trivial; therefore, our approach is limited to the determination of the continuously varying vertical misalignment in the commonly used convergent stereo camera system. This makes the problem more explicit but also simpler to approach since it avoids the use of strong assumptions about the imaging geometry. The advantages include simple implementation and fast rectification by means of image resampling.

In later sections, we describe a procedure that generates a vertically aligned stereo pair for uncalibrated camera arrangements where the assumption that conjugate epipolar lines are collinear is invalid.

\section{EPIPOLAR GEOMETRY}

Conjugate epipolar lines in the images of a stereo pair are collinear to each other (and consequently parallel to the horizontal scan lines of the images) only when the cameras are arranged with their optical axes parallel and perpendicular to the line connecting their optical centers (baseline) and are separated by a simple horizontal shift (a parallel stereo camera system). In any other case, epipolar lines are not collinear, which translates to a nonzero and position-dependent vertical coordinate of disparity. Making implicit use of the epipolar constraint by assuming that the vertical disparity component can be taken as zero can introduce severe errors during the matching process if the stereo camera arrangement is far from parallel.

Fig. 1 illustrates the camera arrangement for convergent stereo. The $X$ axis of the world coordinate system is defined by the baseline. Throughout this section, it is assumed that rotation of either camera 